\title{
OBSERVATION OF VERY LARGE MAGNETIC CONTRAST IN THE SECOND HARMONIC GENERATION FROM Fe(110)
}

\author{
R. Vollmer, M. Straub, and J. Kirschner \\ Max-Planck-Institut für Mikrostrukturphysik \\ Weinberg 2, 06120 Halle/Saale, Germany
}

\begin{abstract}
The nonlinear magneto-optical Kerr effect on a $\mathrm{Fe}(110)$ surface and its dependence on the oxygen exposure is investigated at a wavelength of the incident light of $790 \mathrm{~nm}$ for different geometries. Especially for the transverse Kerr geometry a large intensity change upon reversal of the magnetization direction by a factor more than 4 is observed which can be increased to 80 by oxygen exposure. The magnetization induced part of the nonlinear susceptibility tensor is comparable in size to the nonmagnetic part. KEYWORDS: SECOND HARMONIC GENERATION, MAGNETO-OPTICAL KERR EFFECT, IRON, OXYGEN
\end{abstract}

\section{INTRODUCTION}

Magneto-optical techniques like the magneto-optical Kerr-effect are widely used in the investigation of magnetic properties of materials and in technical applications as well. However, Kerr signals from surfaces and thin films are usually quite small. For example in the transversal Kerr geometry the magnetic contrast, i.e. the ratio of the intensity of the reflected light for the magnetization in opposite directions rarely exceeds several percent. Recently, it was discovered that the frequency doubled light from optical second harmonic generation (SHG) at surfaces or buried interfaces shows generally a much larger magneto-optical Kerr effect $[1,3]$ despite the fact that most of the SHG takes place in an only a few $\AA$ thick layer at the surface or interface. Kerr angles close to $90^{\circ}$ have been reported $[2,4]$. However, these extreme Kerr angles are usually accompanied by a very small average $\mathrm{SH}$ intensity. In this paper we present experimental results which show that magnetic contrast ratios as large as $8000 \%$ are possible in the yield of the second harmonic intensity upon reversal of the magnetization with still considerably high intensity in the average SHG. This is demonstrated on a $\mathrm{Fe}(110)$ surface.

\section{EXPERIMENT}

An elliptically shaped crystal with the long axis parallel to the [001] direction is mounted onto an iron yoke as depicted in Fig. 1. The $F \in(110)$ crystal is cleaned in an UHV chamber (base pressure $4 \times 10^{-11} \mathrm{mbar}$ ) by repeated $500 \mathrm{eV} \mathrm{Ar}{ }^{+}$bombardment at nearly grazing incidence followed by annealing to $850 \mathrm{~K}$. Carbon build up during sputtering was removed by oxygen titration at 750 $\mathrm{K}$. The light of a Ti-sapphire laser at a wavelength of 790 $\mathrm{nm}$ is focused onto the sample and the generated SH light is detected by a photomultiplier. The fundamental laser light was blocked by Schott BGr39 colored glass filters. SH

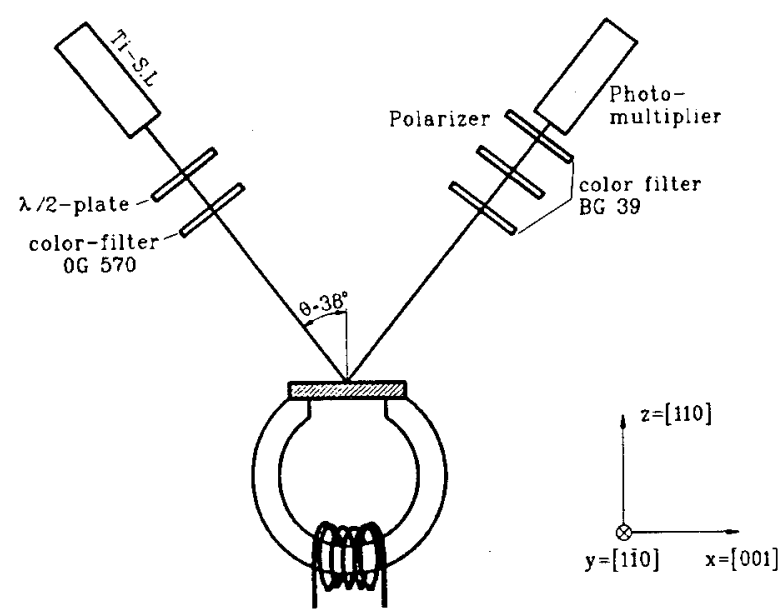

Figure 1: Schematic drawing of the experimental setup. The longitudinal Kerr geometry is shown. For the transverse Kerr geometry the iron yoke with the sample is rotated by $90^{\circ}$ about the surface normal. The sample is magnetized along the [001] direction.

light generated by optical elements in the incident light path was absorbed by an OG570 filter. The iron sample could be magnetized along the [001] direction by running a current of 1 to $3 \mathrm{~A}$ through the coil wound around the iron yoke. By rotating the iron yoke around the crystal normal the direction of the magnetization could be aligned parallel (longitudinal Kerr geometry) or perpendicular (transversal Kerr geometry) to the optical plane. While for the measurements in the longitudinal geometry the polarization of the outgoing SH light was analyzed by a polarizer, the total SH intensity was measured in the experiments with the transversal geometry. In some of the experiments the linear Kerr effect was measured simultaneously by placing a dichroic beam splitter in the 
outgoing beam path. The angle of incidence was about $38^{\circ}$ and measurements were taken at crystal temperatures between $330 \mathrm{~K}$ and $450 \mathrm{~K}$.

\section{THEORY}

The incident laser field generates at the surface and the bulk of the crystal a nonlinear polarization $\mathbf{P}(2 \omega)$, which can be written as:

$$
P_{k}(2 \omega)=\chi_{i j k}^{D} E_{j}(\omega) E_{k}(\omega)+\chi_{i j k l}^{Q} E_{j}(\omega) \nabla_{k} E_{l}(\omega)+\cdots,
$$

with the dipolar and quadrupolar nonlinear susceptibility tensors $\chi_{i j k}^{D}$ and $\chi_{i j k i}^{Q}$. Because of the inversion symmetry of the Fe crystal the first term in eq. (1) vanishes in the bulk and therefore only the surface contributes. Bulk contributions from the second term are always present but are often small compared to the surface contribution of the first, the dipolar term. Quadrupolar contributions to the SH signal will be neglected in the following. The nonlinear polarization $\mathbf{P}(2 \omega)$ gives rise to an outgoing electromagnetic wave with the doubled frequency. The electric fields are the fields inside the medium and therefore the linear optical constants of the $\mathrm{Fe}$ crystal can modify the $\mathrm{SH}$ output. Explicit formulas have been given by a number of authors $[5,6,7,8]$. Without going into detail we note that the elements of the nonlinear susceptibility tensor $\chi_{i j k}^{D}$ can be divided into two classes, elements which are even and elements which are odd with respect to the reversal of the magnetization direction. The measured SH intensity is given then by:

$$
\begin{aligned}
& I_{\uparrow}(2 \omega) \propto\left|\chi_{n m}+\chi_{m}\right|^{2} \\
& I_{1}(2 \omega) \propto\left|\chi_{n m}-\chi_{m}\right|^{2}
\end{aligned}
$$

where $\chi_{n m}$ and $\chi_{m}$ represents a geometry dependent linear combination of tensorelements $\chi_{i j k}^{D}$ including linear optical constants. We will call $\chi_{n m}$ and $\chi_{m}$ the nonmagnetic and the magnetic part of the nonlinear susceptibility, respectively, although $\chi_{n m}$ may contain a magnetization induced fraction as well. $\chi_{m}$ is strictly zero in absence of a magnetization and reverses its sign upon reversal of the magnetization direction. The explicit formulas for the general case are quite complicated. However, for the two geometries considered in this study the situation is similar to the linear case: In the longitudinal geometry, in which the optical plane is parallel to the magnetization direction lying in the surface plane, the s-polarized component of the SH intensity is caused only by magnetic tensorelements $\chi_{m}$ and the p-polarized component only by $\chi_{n m}$ for $\mathrm{p}$-polarized as well as for s-polarized incident light. Therefore, the polarization of the SH light rotates by 2 times the magnitude of the complex Kerr angle:

$$
\tan \Phi_{K}:=\frac{E_{s}(2 \omega)}{E_{p}(2 \omega)}=\frac{\left|\chi_{m}\right|}{\left|\chi_{n m}\right|}
$$

Note the difference to the linear case: Independent of the polarization of the incident light, the nonmagnetic part of
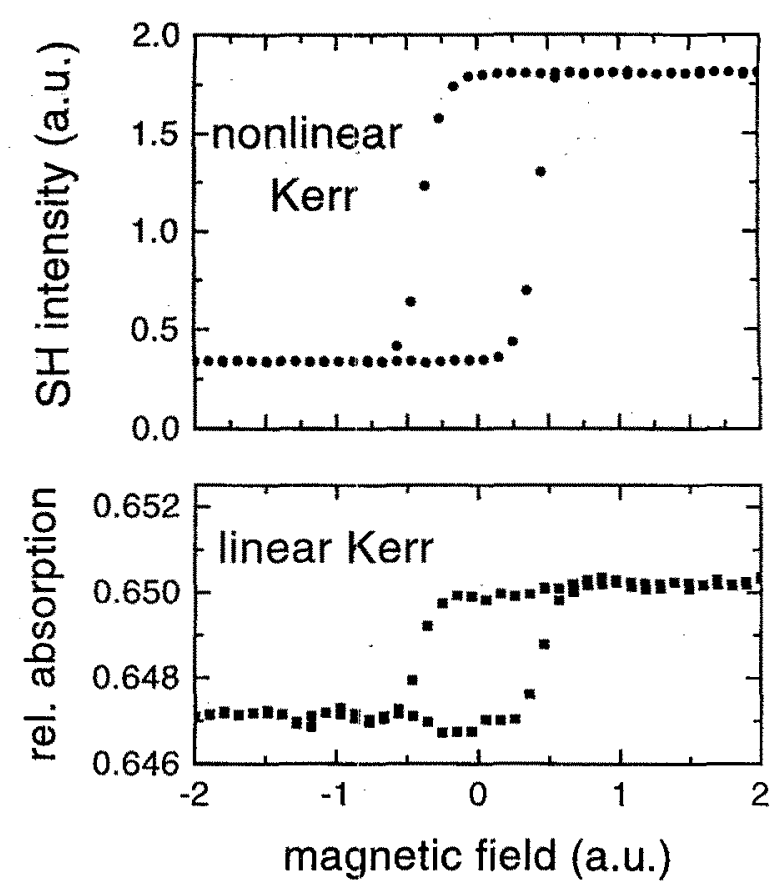

Figure 2: Hysteresis loops from the $\mathrm{Fe}(110)$ crystal taken simultaneously in the transversal Kerr geometry, i.e., the optical plane is along the $\langle 110\rangle$ azimuth. In the upper panel the total SH intensity and in the lower panel the relative absorption of the fundamental light is plotted versus the applied magnetic field.

the SH light is always p-polarized and the magnetic part always s-polarized.

In the transversal geometry all SH light is ppolarized and is generated from magnetic as well as from nonmagnetic components of the susceptibility tensor. No rotation of the polarization axis occurs. Instead an intensity change of the SH light is observed on switching the magnetization direction. The ratio of the intensities can be related to the nonlinear susceptibilities

$$
\frac{I_{\uparrow}(2 \omega)}{I_{\downarrow}(2 \omega)}=\left|\frac{\chi_{n m}+\chi_{m}}{\chi_{n m}-\chi_{m}}\right|^{2} .
$$

Again there is a difference to the linear case: Even for s-polarized incident light a nonlinear Kerr effect is not forbidden by symmetry rules.

Including bulk contributions would not change the above considerations for the geometries considered. Merely a redefinition of $\chi_{n m}$ and $\chi_{m}$ is necessary now including tensorelements of $\chi_{i j k l}^{Q}$ besides those of $\chi_{i j k}^{D}$.

\section{RESULTS}

Figure 2 shows two hysteresis loops taken simultaneously in transversal geometry. In the top panel the SH intensity and in the bottom panel the relative absorption of the incident light is shown as a function of the applied magnetic field. While the relative intensity change of the 
reflected light, which is the linear transverse Kerr signal, is only of the order of $0.5 \%$ the $\mathrm{SH}$ intensity changes by more than a factor of 5 upon reversal of the magnetization direction. This extreme contrast ratio can be enhanced further quite dramatically by oxygen adsorption as depicted in Fig. 2. The SH intensity for the magnetization in opposite directions, $I_{\uparrow}$ and $I_{\downarrow}$, as well as the arithmetic average $\mathrm{SH}$ intensity (dashed line) is plotted in the bottom panel, and the contrast ratio $I_{\uparrow} / I_{\downarrow}$ in the upper panel as a function of the oxygen exposure. The incident light was p-polarized. Up to several 10 Langmuir (L) of oxygen the the average SH intensity remains more or less constant except a region around 3 to $4 \mathrm{~L}$ exposure where the formation of an ordered $c(2 \times 2)$ oxygen superstructure enhances the average intensity by a factor of nearly two. However, the magnetic contrast ratio shows little variation until around 30 to $40 \mathrm{~L}$ it reaches extreme values up to 80 . The oxygen coverage has been determined

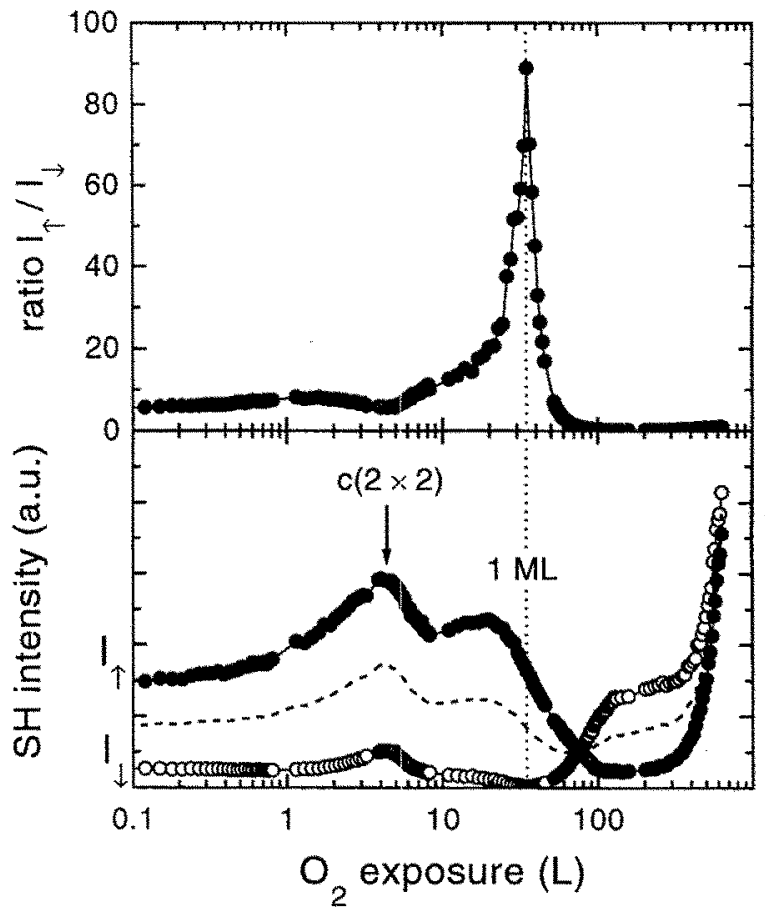

Figure 3: Total second harmonic intensity from a $\mathrm{Fe}(110)$ surface for the transversal Kerr geometry as a function of the oxygen exposure for p-polarized incident light. Bottom panel: $I_{\uparrow}$ (filled symbols) and $I_{\downarrow}$ (open symbols) denote the intensity for the magnetization in opposite directions. The dashed line represents the arithmetic average of $I_{\uparrow}$ and $I_{\downarrow}$. Upper panel: The magnetic contrast ratio $I_{\uparrow} / I_{\downarrow}$ calculated from the intensities of the bottom panel. Crystal temperature was about $330 \mathrm{~K}$.

by Auger spectroscopy and low energy electron diffraction (LEED) and compared to the results of Miyano et al. [9]. While the coverage up to $1 / 4 \mathrm{ML}$ can be calibrated quite precisely by that exposure where the $c(2 \times 2)$ superstructure is fully developed, the higher coverage are less accurately determined. This is mainly due to the following two reasons: At coverage higher than $0.4 \mathrm{ML}$ oxide formation sets in making the coverage determination by auger spectroscopy less reliable. In addition, while the sticking coefficient is nearly temperature independent up to $1 / 4 \mathrm{ML}$ coverage it becomes strongly temperature dependent above [10]. Due to these reasons the exposure for nominally $1 \mathrm{ML}$ coverage is determined only within a factor of $2-3$ and occurs around 30 to $40 \mathrm{~L}$ at a crystal temperature of $330 \mathrm{~K}$.

To demonstrate, that the contrast ratio depends strongly on the incident polarization, in Fig. 4 the SH intensity for the magnetization in opposite directions as well as the contrast ratio $I_{\uparrow} / I_{\downarrow}$ is shown for s-polarized incident light. Unlike for p-polarized incident light seen in

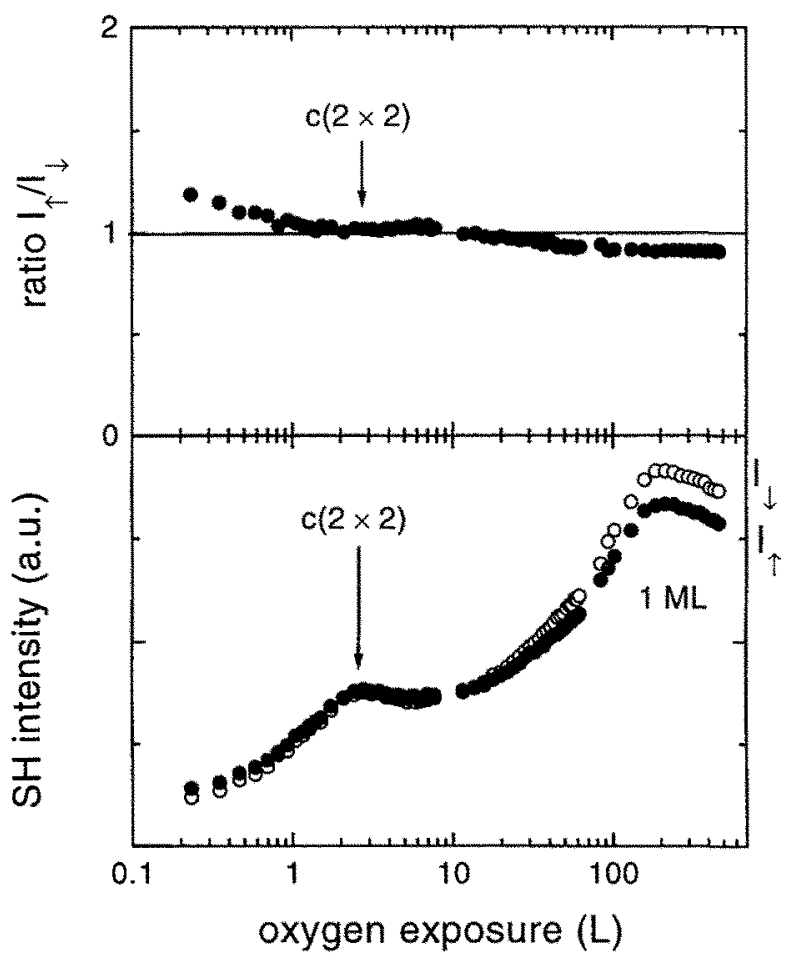

Figure 4: Same as Fig. 3 for s-polarized incident light. Crystal temperature was about $470 \mathrm{~K}$.

Fig. 3, the SH intensity increases for s-polarized incident light strongly with the oxygen coverage but the contrast ratio $I_{\uparrow}$ and $I_{\downarrow}$ remains always close to 1 . At $1 / 4$ coverage the contrast almost vanishes. The contrast reverses at coverage much below $1 \mathrm{ML}$. (Note that due to the higher crystal temperature $1 \mathrm{ML}$ coverage is reached at about $200 \mathrm{~L}$.) We remember that the nonlinear Kerr effect for s-polarized incident light in the transverse geometry does not vanish by symmetry rules in contrast to the linear Kerr effect.

In the longitudinal geometry no change in the total intensity is observed due to oxygen exposure. As discussed above in this geometry the magnetization causes a rotation of (and introduces an ellipticity in) the polar- 
ization of the outgoing SH light. By placing an analyser in the outgoing beam path the polarization ellipse can be measured as it is shown in Fig. 5. For p-polarized inci-

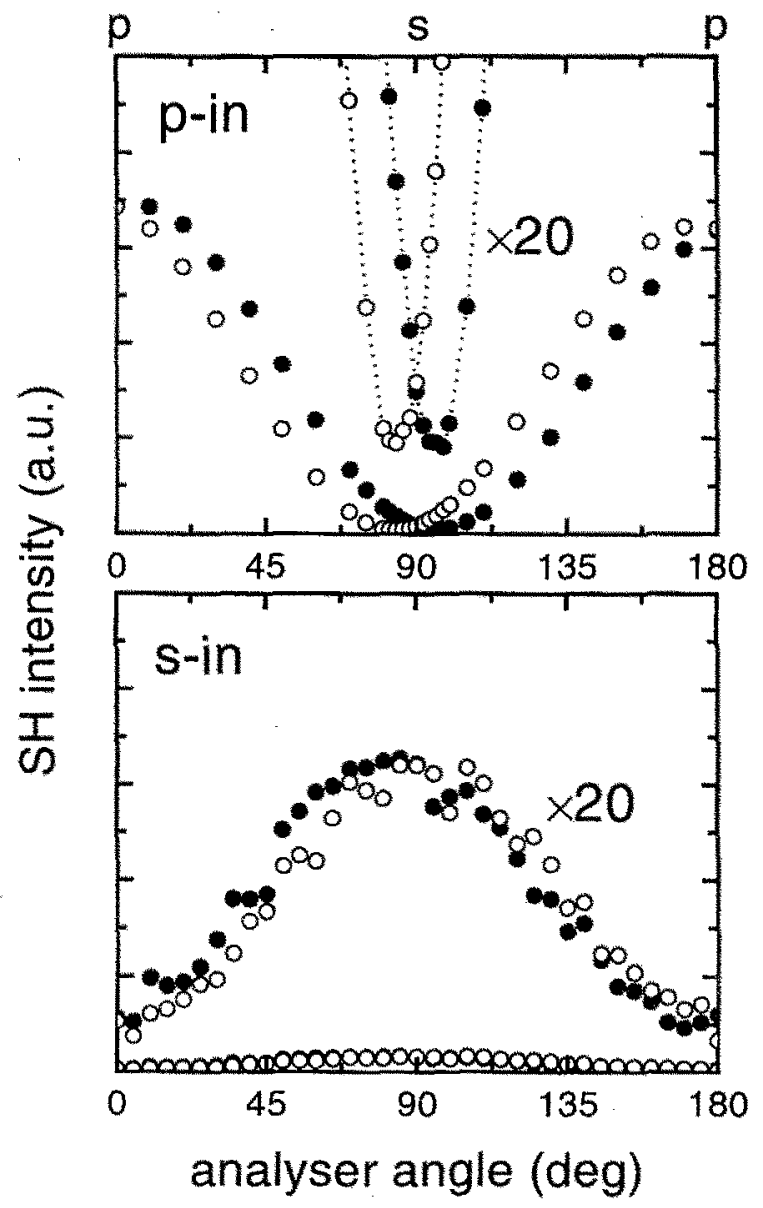

Figure 5: SH intensity from the clean Fe(110) surface in the longitudinal geometry as a function of the polarizer angle $\alpha$. Top panel for p-polarized incident light, bottom panel for $s-$ polarized incident light. $I_{\uparrow}$ (filled symbols) and $I_{\downarrow}$ (open symbols) denote the intensity for the magnetization in opposite directions. $\alpha=0$ corresponds to p-polarized outgoing $\mathrm{SH}$ light, $\alpha=90^{\circ}$ to s-polarized SH light.

dent light a maximum contrast ratio of about 5 is obtained at an analyser angle $\alpha=80^{\circ}$ but the average SH intensity is only $1 / 10$ of the maximum intensity at $\alpha=0^{\circ}$. The magnitude of the complex Kerr angle as defined in eq. (3) is calculated from the SH intensity curves in Fig. 5 and amounts to $\Phi_{K}=10^{\circ}$. The background signal is almost ten times lower than the minimum signal measured. Therefore, the relatively low contrast ratio is caused by the ellipticity change of the SH light. Decomposing the (complex) Kerr angle into a pure rotation and an ellipticity results in $7^{\circ}$ for both, the rotation and the ellipticity change. For s-polarized incident light the p-polarized SH intensity at $\alpha=0^{\circ}$ is close to zero while there is definitely an s-polarized SH signal at $\alpha=90^{\circ}$. From the symmetry analysis it is known that the s-polarized SH intensity is entirely due to the magnetic part of the nonlinear susceptibility and consequently a Kerr rotation close to $90^{\circ}$ occurs. However, the magnetic contrast is almost zero for all analyser angle because of the missing nonmagnetic part. The latter part can be enhanced by oxygen adsorption reducing the magnetic part only moderately. The Kerr angle is reduced to about $20^{\circ}$ but the magnetic contrast increases up to 10 .

The above example shows that in the case of the nonlinear Kerr effect the Kerr angle as well as the magnetic contrast ratio $I_{\uparrow} / I_{\downarrow}$ are by themselves not good measures of the size of the magnetization induced SHG because of the large variation of the nonmagnetic part. The magnetic and nonmagnetic parts of the nonlinear susceptibility can be measured separately at analyser angles of $0^{\circ}$ and $90^{\circ}$, respectively, for the longitudinal geometry. Figure 6 gives an overview of the SH intensity for all four polarization combinations of the incident light and the outgoing SH light. The s-polarized outgoing SH intensity is proportional to $\left|\chi_{m}\right|^{2}$ and the p-polarized SH proportional to $\left|\chi_{n m}\right|^{2}$. (Note, that the intensity is proportional

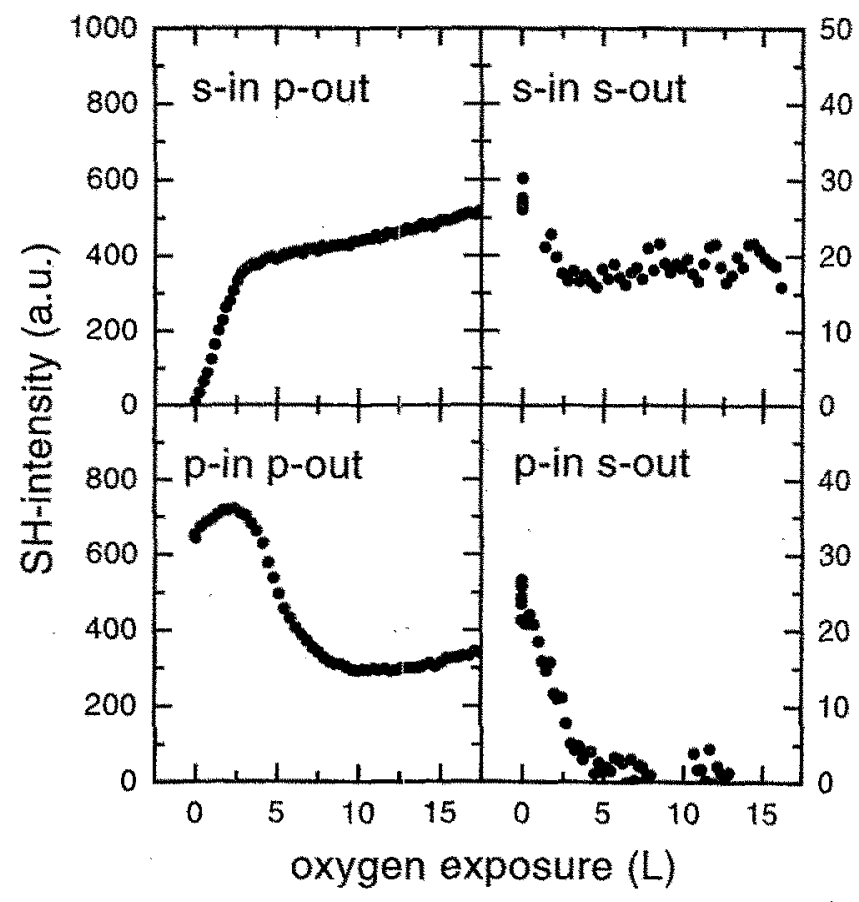

Figure 6: SH intensity as a function of the oxygen exposure for all four combinations of the polarization of the incident fundamental light and the outgoing SH light. The SH intensity in the left two panels is generated by the nonmagnetic tensorelements $\chi_{n m}$ and the SH intensity in the right two panels is generated by the magnetic tensorelements $\chi_{m}$. Crystal temperature was $470 \mathrm{~K}$.

to the square of the nonlinear susceptibility.) Therefore, the magnetic part of the susceptibility is comparatively little influenced by oxygen adsorption for s-polarized incident light. For p-polarized incident light $\chi_{m}$ drops by a 
much larger amount but it becomes definitely not zero as there is clearly a magnetic contrast observable at analyser angles other than $90^{\circ}$ for the whole investigated range of oxygen exposures. We also note, that the nonmagnetic part of the SH intensity does not drop dramatically on oxygen exposure. For s-polarized incident light instead it increases from nearly zero to a value exceeding that for the p-polarized SH intensity.

\section{DISCUSSION AND CONCLUSION}

The experimental results presented in the previous section clearly show, that the nonlinear Kerr effect exceeds the linear Kerr effect by orders of magnitude for all geometries investigated. The much enlarged nonlinear Kerr angles were predicted theoretically by Hübner and Bennemann [11] and have been demonstrated experimentally on thin films $[3,7,12,13]$ and surfaces $[1,2,6,14]$. In all cases a large nonlinear Kerr effect is found compared to the linear case. However, only the studies of Cobalt films on $\mathrm{Cu}(001)[3,13]$ have been performed under UHV conditions with well characterized surfaces. There, in the transversal Kerr geometry a magnetic contrast of about 2 has been observed for the clean Co films using p-polarized incident light. Adsorption of oxygen or CO increased this contrast up to more than 5. As the nonlinear Kerr signal was independent of the film thickness, ruling out significant bulk contributions to the Kerr signal, the magnetic SH light is generated predominantly at the surface and the buried $\mathrm{Co} / \mathrm{Cu}$ interface. Because reactive gases like oxygen tend to decrease the magnetization at the surface it seems quite reasonable to assume a reduction of the magnetic $S H$ signal from the surface by oxygen exposure. Because of the opposite sign of the nonlinear susceptibility tensor elements at the surface and interface the destructive interference of the two contributions is lifted and an increased Kerr signal results.

In the present case, however, there is only one interface, the surface, contributing to the SH signal and consequently the situation discussed above does not apply. As bulk contributions may no more be negligible in contrast to the thin film case, the superposition of bulk contributions and surface contributions can also lead to an increased magnetic contrast in the transversal geometry. But this is not the only possible explanation for the increased magnetic contrast with increasing oxygen exposure. At the peak of the magnetic contrast in Fig. 2 the magnetic contributions $\chi_{m}$ and the nonmagnetic contributions $\chi_{n m}$ are very similar in size as can be derived from eq. (4). Therefore, it is not a priori clear if $\chi_{n m}$ is larger than the magnetization induced part $\chi_{m}$. Instead assuming $\left|\chi_{m}\right|>\left|\chi_{m m}\right|$ would lead to an increased magnetic contrast with decreasing magnitude of $\chi_{m}$. In this case $\left|\chi_{m}\right|$ of the clean Fe(110) surface would be at least. 2.6 times the magnitude of $\left|\chi_{n m}\right|$. It can be even larger because $\chi_{m}$ as well as $\chi_{n m}$ are complex numbers and the phase between them is not known from our measurements.
Independent of any assumption the minimum ratio $\left|\chi_{m} / \chi_{n m}\right|$ must be $\geq 0.4$ for the clean Fe(110) surface and for the oxygen covered surface at the maximum peak of the magnetic contrast $\geq 0.8$. Because of the large number of contributing tensorelements $\chi_{i j k}^{D}$ (even when neglecting the contributions from $\chi_{i j k l}^{Q}$ ) a determination of the magnitude (and relative phase) of the individual tensorelements is not possible from our present measurements. The situation is much simpler for s-polarized incident light (Fig, 4). Here, only one nommagnetic $\chi_{z x x}^{D}$ and one magnetic tensorelement $\chi_{y x x}^{D}$ contribute. As seen in Fig. 4 the magnetic contrast is quite low, 1.3 for the clean Fe(110) surface. Taking the linear optical constants into account (refractive index $N(2 \omega)=1.98+3.18 i$ ) results in a lower limit of $\chi_{y x x}^{D} / \chi_{z x x}^{D} \geq 0.15$ which is quite significant. The relatively low magnetic contrast obtained in this case is caused mainly by the enhancement of the nonmagnetic tensorelement, $\chi_{i j k}^{D}$, by the Fresnel coefficient, i.e., the geometry. Smaller incident angles would result in an increased magnetic contrast.

Complete information about the size and the relative phase of the contributing tensorelements $\chi_{i j k}^{D}$ can be obtained from measurements in the longitudinal geometry. For s-polarized incident light again only one magnetic, $\chi_{x x x}^{D}$, and one nonmagnetic tensorelement, $\chi_{z x x}^{D}$, contribute. From the relative intensities in Fig. 6 a ratio $\left|\chi_{x x x}^{D}\right| /\left|\chi_{z x x}^{D}\right|=0.6$ can be derived for the $c(2 \times 2)$ oxygen superstructure at about $3 \mathrm{~L}$ oxygen exposure. At this coverage the nonmagnetic part of the SH intensity is not much lower than the maximum intensity for $p$ polarized incident light. (The phase between $\chi_{x: x}^{D}$ and $\chi_{z x x}^{D}$ is about $89^{\circ} \pm 5^{\circ}$.) For the clean $\mathrm{Fe}(110)$ surface the ratio $\left|\chi_{x w x}^{D}\right| /\left|\chi_{z x: x}^{D}\right|$ becomes very large only because the nonmagnetic SH intensity is almost zero. For p-polarized incident light three nonmagnetic and two magnetic tensorelements contribute to the SH signal so that a determination of the magnitude of individual tensorelements are not possible with the present data.

Above we have discussed the SHG only in terms of the dipolar surface term $\chi_{i j k}^{D}$. Very likely, the bulk contributes to the $\mathrm{SH}$ yield due to $\chi_{i j k l}^{Q}$ as it has been found for other systems [16]. A clear separation between these two contributions by adsorption experiments is not uniquely possible. As we have shown in this paper oxygen adsorption does not simply cancel or reduce the $\mathrm{SHG}$ from the surface. Instead we observe a maximum in the $\mathrm{SH}$ intensity at the formation of the ordered $c(2 \times 2)$ superstructure. After the onset of oxide formation further complications arise from the change in the surface morphology [15]. The strong increase of the average SH intensity in Fig. 3 for exposures above $400 \mathrm{~L}$ may be caused by the formation of small iron oxide clusters.

In summary we have shown that magnetization induced SHG is of the same order of magnitude as the nonmagnetic part. For the transverse Kerr geometry this ratio can be tuned to a value close to 1 by oxygen ex- 
posure resulting in a very large magnetic contrast up to 80. The maximum contrast in the longitudinal geometry is limited to values about 10 or lower due to considerable magnetization induced ellipticity in the outgoing SH light.

[1] J. Reif, J.C. Zink, C.M. Schneider, and J. Kirschner, Phys. Rev. Lett. 67, 2878 (1991).

[2] J. Reif, C. Rau, and E. Matthias, Phys. Rev. Lett. 71, 1931 (1993).

[3] H.A. Wierenga, W. de Jong, M.W.J. Prins, Th. Rasing, R. Vollmer, A. Kirilyuk, H. Schwabe, and J. Kirschner, Phys. Rev. Lett. 74, 1462 (1995).

[4] B. Koopmans, M. G. Koerkamp, Th. Rasing, and H. van den Berg, Phys. Rev. Lett. 74, 3692 (1995).

[5] J.E. Sipe, D.J. Moss, and H.M. van Driel, Phys. Rev. B 35,1129 (1987).

[6] K. Böhmer, J. Hohlfeld, and E. Matthias, Appl. Phys. 60, 203 (1995).

[7] B. Koopmans, A.M. Janner, H.A. Wierenga, Th. Rasing, G.A. Sawatzky, and F, van der Woude, Appl. Phys. 60, 103 (1995).

[8] W. Hübner and K.H. Bennemann, to be published.

[9] T. Miyano, Y. Sakisaka, T. Komeda, and M. Onchi, Surf. Sci. 169, 197 (1986).

[10] V.S. Smentkowski and J.T. Yates, Jr. Surf. Sci. $232,113(1990)$.

[11] W. Hübner and K.H. Bennemann, Phys. Rev. B 40, 5973 (1989); W. Hübner, Phys. Rev. B 42, 11553 (1990); U. Pustogowa, W. Hübner, K.H. Bennemann, Phys. Rev. B 48, 8607 (1993);

[12] H.A. Wierenga, M.W.J. Prins, D.L. Abraham, and Th. Rasing, Phys. Rev. B 50, 1282 (1994).

[13] R. Vollmer, A. Kirilyuk, H. Schwabe, J. Kirschner, H.A. Wierenga, W. de Jong, M.W.J. Prins, and Th. Rasing, J. Magn. Magn. Mater. 148, 295 (1995).

[14] R. Vollmer, M. Straub, and J. Kirschner, accepted for publication in Surf. Sci.

[15] A. Wight, N.G. Condon, F.M. Leibsle, G. Worthy, A. Hodgson, Surf. Sci. 331 - 333, 133 (1995).

[16] R. Vollmer, M. Straub, and J. Kirschner, accepted for publication in Surf. Sci. 